\title{
LE REFROIDISSEMENT ET LA CONGÉLATION DU LAIT
}

par M. Henri CORBLIN, Ingénieur agronome.

(Suite et fin)

Lait de ramassage. - Pour des quantités importantes, on peut procéder de deux façoñs :

a) Avoir, lorsque cela est possible, des centres de ramassage munis d'un petit appareillage frigorifique ; dans ces centres et dépôts, plusieurs cultivateurs apporteront leur lait sitôt la traite; ce lait sera mis par le dépositaire immédiatement dans le bain de saumure, et les bidons, une fois retirés, et après immersion rapide dans l'eau, seront conservés dans un coffre ou un meuble bien isolé jusqu'au passage de la voiture qui les emportera à la gare voisine ou qui les distribuera en ville ;

b) On peut conserver les usines actuelles de pasteurisation dans lesquelles, après passage dans ou sur les appareils, on refroidira le lait du mieux possible avec l'eau courante; on plongera ensuite les bidons une vingtaine de minutes dans le bain de saumure à très basse température. En procédant ainsi, qu'il s'agisse de lait pasteurisé ou non, on prolonge de beaucoup la durée de conservation de ce lait; en conséquence, on facilite son transport et on peut étendre à de longues distances les rayons de ramassage des grandes villes.

Que l'on ait affaire à un petit nombre de bidons ou à une exploitation industrielle, il faut évidemment orienter le travail de telle façon que les bidons ne restent pas plus de temps qu'il ne convient dans la saumure, en partant du principe que la congélation doit être très active et se faire en couche mince pour que le lait congelé soit de composition homogène. Les pots ainsi préparés sont, comme il a déjà été dit, placés dans un coffre, dans un meuble, ou dans une chambre, isolés de la chaleur jusqu'au moment de leur expédition.

On peut, en opérant de cette façon, conserver du lait maintenu à une température proche de $0^{\circ}$ une vingtaine de jours sans que son acidité augmente et sans que son apparence change, mais il ne faut pas oublier que certains germes ou microbes travaillent et se multiplient à basse température, et c'est pourquoi, à partir du début de la troisième semaine, la saveur du lait ainsi conservé se modifie peu à peu. Ce temps de conservation est d'ailleurs, en fait, inutile et, dans la pratique courante, il ne s'écoule que quelques jours entre le moment de la traite et celui où le lait est consommé. On obtient à coup sûr ce résultat en procédant comme je viens de l'indiquer.

On objectera que, dans une ferme et pour de petites quantités, le refroidissement, par la saumure, du lait sortant du pis jusqu'à une température voisine de celle de l'eau courante dont on dispose, est plus coûteux que le refroidissement direct par cette eau courante, mais 
c'est une objection purement théorique, car il s'agit-là d'une dépense de 3 ou $4 \mathrm{fr}$. de force motrice pour quelques centaines de litres de lait, et cette somme est certainement plus faible que le coût de la maind'œuvre necessitée par le nettoyage et la stérilisation des bacs, robinets, réfrigérants, etc., ainsi que du temps passé pour le réglage des débits et la surveillance lorsqu'on opère avec l'eau. De plus, tous transvasements et manipulations subis par le lait ne peuvent lui être que défavorables. Il ne faut pas oublier que la température de 16 ou $18^{\circ}$ obtenue pour le lait au moyen de l'eau courante est tout à fait insuffisante pour une bonne conservation dans les jours chauds, tandis que la couche protectrice de lait congelé sur la paroi du bidon, en plus du refroidissement du liquide au voisinage de $0^{\circ}$, évite une élévation de la température du lait, surtout contre la partie de la paroi pouvant être exposée au soleil au cours du transport, laquelle étant portée à une température relativement élevée provoque, dans le liquide, une multiplication très rapide des germes.

Lorsqu'on opère sur un certain nombre de bidons, le poids total de lait congelé dans ces bidons est suffisant pour maintenir la conservation dans un coffre ou une armoire bien isolée ou même un wagon isotherme, sans qu'il soit besoin de glace, mais il est clair qu'un récipient tout seul, mis à l'air libre, perd proportionnellement plus de froid que des bidons groupés. Il suffit, pour transporter un lot de bidons préparés comme il est dit plus haut, de le protéger par des couvertures ou des matelas isolants appropriés.

Si l'on apporte dans la traite toutes les précautions hygiéniques possibles, et si l'on veut traire sur du lait congelé, il est facile de le faire car le bac à saumure est, comme il a été expliqué, plus grand qu'il n'est nécessaire pour contenir les bidons de traite et l'on peut, en dehors de quelques mouleaux de glace qui y trouvent toujours place, conserver des bidons dans lesquels on a formé une couche de lait congelé contre les parois; ees bidons étant vides de liquide, on les emploiera d'une traite à la suivante, de telle manière qu'au sortir du trayon, le lait se trouvera immédiatement refroidi. Ces conditions assurent le maximum de conservation si l'on maintient les bidons au voisinage de $0^{\circ}$, mais dans la pratique, on constate qu'il n'est pas absolument nécessaire de traire sur du lait congelé pourvu qu'on refroidisse immédiatement et énergiquement après la traite, car les colonies microbiennes ne se sont pas encore développées.

Les figures 1 et 2 correspondent à deux types d'installations : l'une pouvant traiter quelques centaines de litres de lait, la seconde quelques milliers de litres.

Dans la figure 1 , on voit le compresseur et le conderseur de la machine frigorifique ainsi que le bac à saumure dans lequel sont plongés les 


\section{Sefroidissement et congélation an Rait.}

\section{Breveto Partix.}
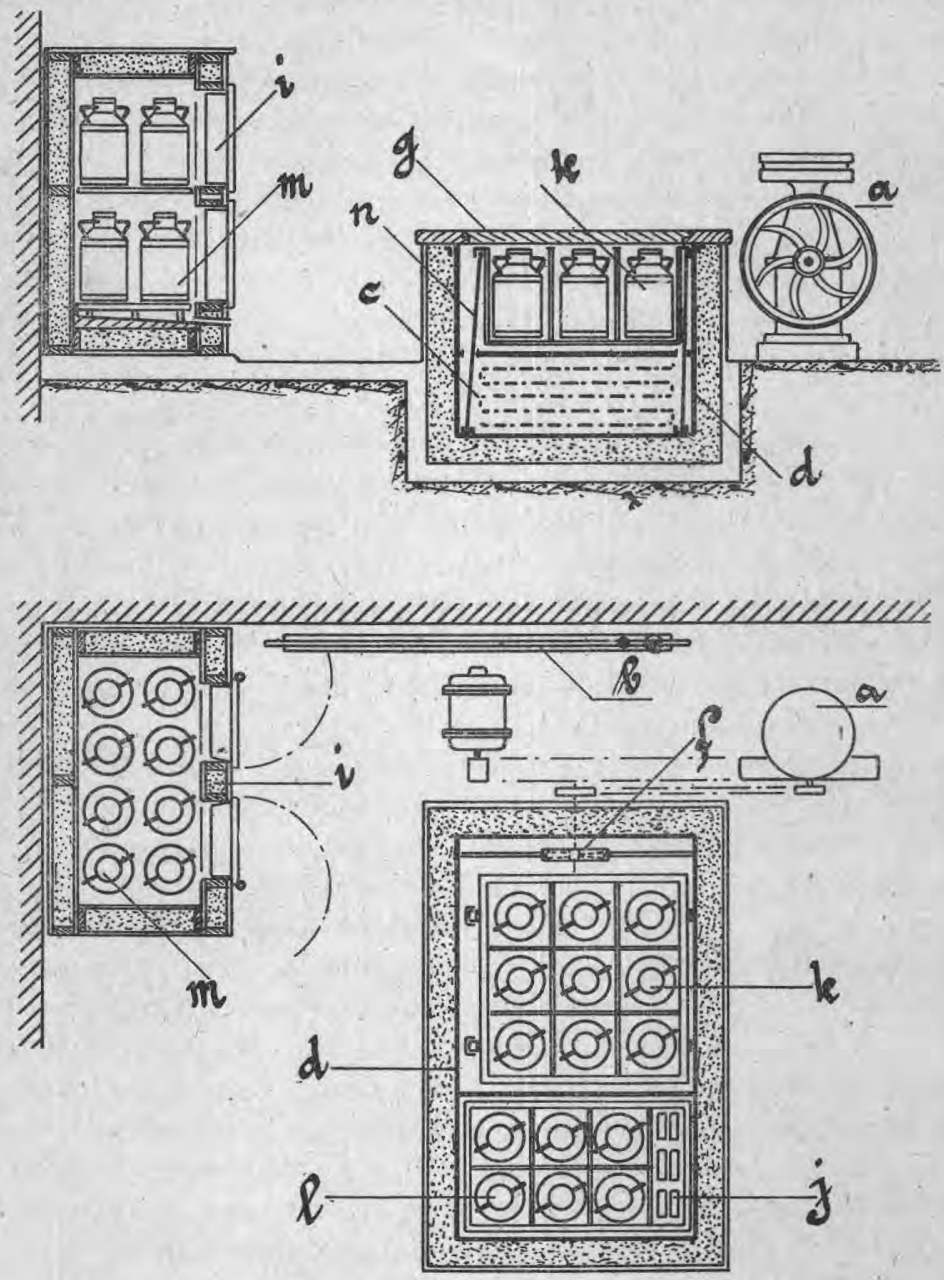

Fig. 1. - Installation pour le refroidissement et la congélation de quelques centaines de litres de lait.

a) - Compresseur à membrane Corblin

b) - Condenseur à contre-courant

c) - Serpentin évaporateur

d) - Bac à saumure

f) - Hélice d agitation de saumure

g) - Couvercle du tac

i) - Armoire isolée pour pots de lait $m$.

) - Mouleaux à glace k) - Pots à lait avec une enveloppe de lait en cours de congélation

l) - Pots a lait prêts à recevoir une traite dans ıeur enveloppe congelée

m) Pots à lait en attente de 1 'expédition

$n$ ) - Chassis articulé supportantle cageot mobile: contenant les pots $k$ 
bidons; sur ceux-ci, un couvercle est rabattu pour les maintenir convenablement immergés et éviter les pertes de froid. Une hélice agite vivement la saumure qui circule sur les serpentins réfrigérants et tout autour des bidons. Dans ce bac se trouvent également quelques mouleaux servant aे la fabrication de la glace et des bidons ayant leurs parois recouvertes de lait congelé, si l'on désire traire directement dans des bidons ainsi préparés.

La figure 2 montre une machine frigorifique plus importante avec son compresseur, son condenseur et un frigorifère de grand volume; un pont-roulant monté au-dessus de ce frigorifère permet à un cageot, contenant par exemple une vingtaine de bidons, de recevoir ceux-ci lorsqu'il repose sur le sol, puis ce cageot, garni et muni de son couvercle, est plongé pendant une vingtaine de minutes dans le bain de saumure très froide et vivement agitée. Ce même cageot est retiré de la saumure au moyen du pont-roulant, puis trempé rapidement dans un bain d'eau qui enlève la pellicule de saumure restant sur les bidons : il est tiré aussitôt de ce bain et finalement débarrassé des bidons qui sont ainsi prêts pour le transport. On garnit à nouveau le cageot et l'opération recommence.

La congélation du lait est utile lorsqu'il s'agit de lui faire subir de longs transports ou bien de lui conserver toutes ses qualités chez le détaillant et chez le consommateur - si ce dernier veut bien en prendre soin - mais elle n'est pas toujours indispensable, par exemple pour livrer en ville le lait d'une ferme voisine ou distante de 10 ou $20 \mathrm{~km}$., ce qui est facile si le transport se fait en camionnette automobile. La livraison peut ne s'effectuer qu'une fois par jour : c'est le plus souvent le matin; il faut donc dans ce cas conserver la traite de la veille au soir, refroidir rapidement celle qui vient d'être faite et distribuer en une fois le produit des deux traites, soit dans les boutiques des détaillants, soit directement aux particuliers.

On peut obtenir de bons résultats sans congélation en refroidissant à basse température, dans les bidons de transport, le lait sitôt trait, évitant ainsi les transvasements et les manipulations toujours nuisibles. L'eau glacée suffit pour cela (fig. 3).

On place les bidons dans un bac rempli d'eau qui est maintenue à $0^{\circ}$, soit par l'addition d'une quantité de glace suffisante, soit par un petit appareil frigorifique doht le serpentin frigorifère est confectionné spécialement pour que, disposées dans le bac à eau, les spires de ce serpentin s'entourent, pendant toute la durée du fonctionnement de la machine, d'un manchon de glace qui sert d'accumulateur de froid et qui fond plus ou moins lorsqu'on plonge, dans ce même bac, les bidons contenant le lait encore chaud de la traite.

Pour obtenir un refroidissement énergique, il convient de disposer 


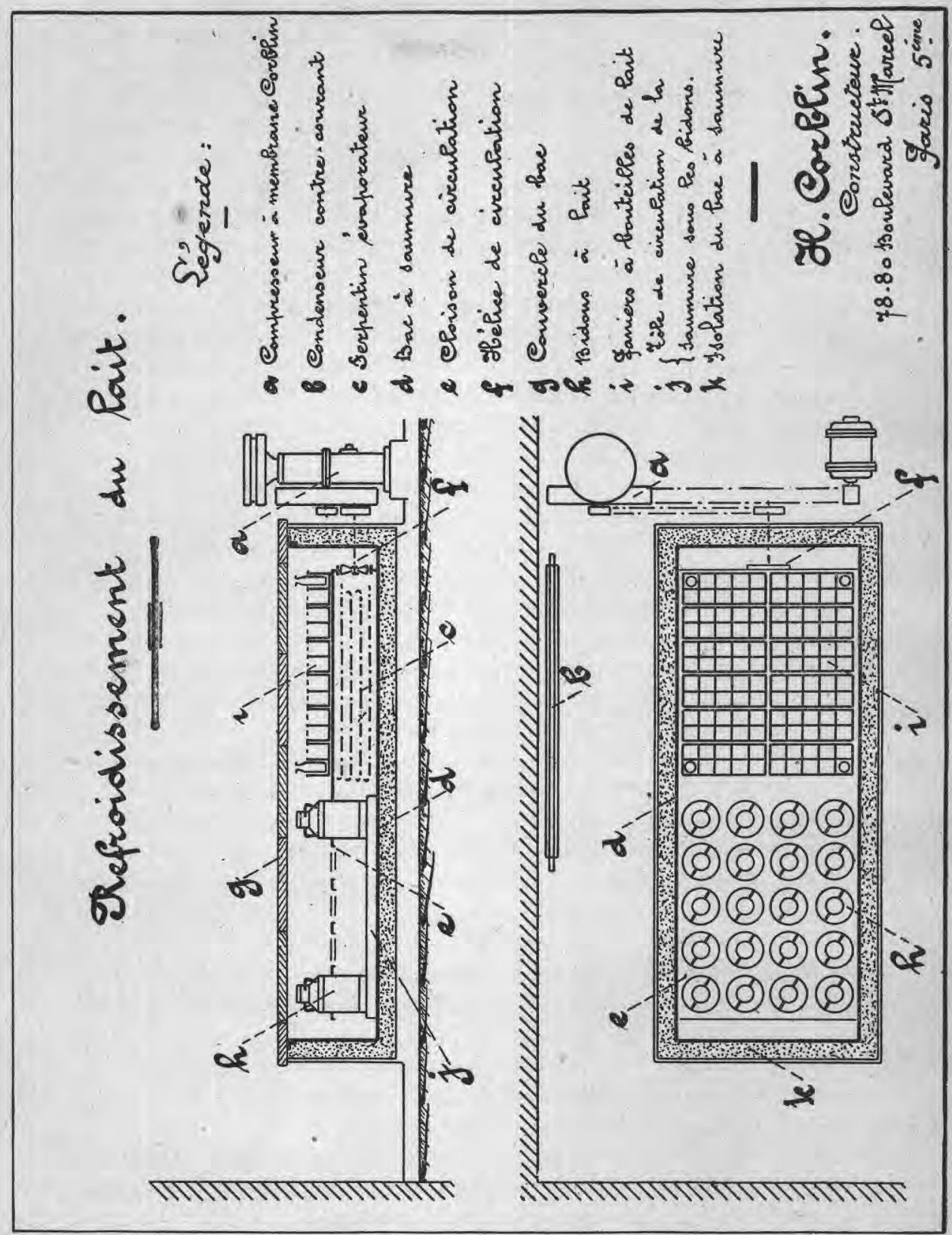

Fig. 3. - Installation pour e refroidissement sans congélation de quelques centaines de litres de lait. 
dans le bac une hélice d'agitation de l'eau glacée avee une cloison de circulation, ce qui permet de faire passer sur les bidons un rapide courant d'eau, en même temps que s'opère la fusion de la glace qui maintient cette eau à $0^{\circ}$. Dans ces conditions, la température du lait s'abaisse vivement et l'ensemencement microbien ne se produit pas. En moins d'une heure, la température du lait d'un bidon de 20 litres descend, sans qu'il soit remué, à environ $4^{\circ}$ et continue naturellement à se rapprocher de $0^{\circ}$ si le lait reste dans le bac. On conserve de cette façon le lait de la traite du soir en laissant les bidons dans le bain d'eau glacée, et même si la chose est nécessaire, en raison des exigences du transport st. de la livraison, on peut très bien garder la traite du matin d'un jour sur l'autre sans craindre aucune altération, ce qui donne beaucoup de facilité pour choisir au mieux les heures de traite et de départ de la voiture.

Lorsque la ville proche possède une fabrique de glace qui peut livrer régulièrement et à coup sûr, en même temps qu'à un prix assez bas, la quantité de glace qui est chaque jour nécessaire, la voiture qui porte le lait prendra cette glace directement à l'usine à son retour.

Pour fixer les idées et comparer le coût de la glace produite par un petit appareil frigorifique installé dans la ferme, fonctionnant sur le bac à eau glacée, avec le prix de la glace payé à l'usine, on peut se baser sur les chiffres suivants.

Les facteurs entrant dans le prix de revient de la glace accumulée sur les serpentins du bac sont: la force motrice, l'amortissement $\mathrm{du}$ matériel et la surveillance. C'est surtout la force motrice qui domine, car l'amortissement est insignifiant s'il s'agit par exemple de 200 à 500 litres de lait par jour, et la surveillance, dans la plupart des cas, n'est pour ainsi dire pas à compter; il en est de même pour les frais. d'entretien qui restent peu élevés.

Si le courant électrique coûte 1,50 fr. le kilowatt, on peut acheter la glace $9 \mathrm{fr}$. les $100 \mathrm{~kg}$. au maximum; si le courant coûte seulement I fr. ou bien si la force motrice revient encore à un prix plus bas, il ne faut pas payer la glace plus de 6 à $6,50 \mathrm{fr}$. les $100 \mathrm{~kg}$.

Lorsque le prix de vente de la glace est plus élevé, il y a intérêt à installer un patit appareil frigorifique. Il est évident que, dans tous les cas où l'on ne peut pas se procurer facilement et à coup sûr les quantités. de glace nécessaires, l'installation frigorifique s'impose.

La figure 3 montre le schéma d'une telle installation. On voit dans. le bac à eau glacée, non seulement le serpentin et l'hélice d'agitation, les bidons en cours de refroidissement, mais encore les paniers dans lesquels se trouvent les bouteilles pour le cas où on livre le lait directement aux consommateurs. On comprend qu'il est utile de refroidir ces bouteilles. avant de les remplir de lait. Celui-ci étant à une température voisine de $0^{\circ}$, il faut que le récipient, surtout s'il est en verre épais, soit ramené à la même température avant son remplissage. 